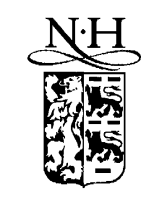

ELSEVIER

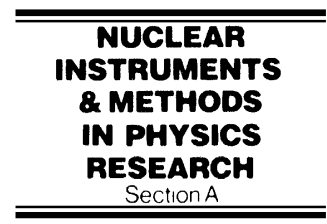

www.elsevier.com/locate/nima

\title{
Discharge studies and prevention in the gas electron multiplier (GEM)
}

\author{
S. Bachmann ${ }^{\mathrm{a}}$, A. Bressan ${ }^{\mathrm{a}}$, M. Capeáns ${ }^{\mathrm{c}}$, M. Deutel ${ }^{\mathrm{a}}$, S. Kappler ${ }^{\mathrm{b}}$, \\ B. Ketzer ${ }^{\mathrm{a}}$, A. Polouektov ${ }^{\mathrm{d}}$, L. Ropelewski ${ }^{\mathrm{a}}$, F. Sauli ${ }^{\mathrm{a}, *}$, E. Schulte ${ }^{\mathrm{e}}$, \\ L. Shekhtman ${ }^{\mathrm{d}}$, A. Sokolov ${ }^{\mathrm{d}}$

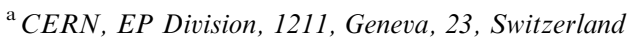 \\ ${ }^{\mathrm{b}}$ Institut für Experimentelle Kernphysik, Karlsruhe University, Germany \\ c Physikalisches Institut, Heidelberg University, Germany \\ ${ }^{\mathrm{d}}$ Budker Institute for Nuclear Physics, Novosibirsk, Russia \\ ${ }^{\mathrm{e}}$ Institute of Physics, Helsinki University, Finland
}

Received 18 December 2000; received in revised form 29 January 2001; accepted 5 March 2001

\begin{abstract}
The gas electron multiplier (GEM) used as single proportional counter or in a cascade of two or more elements, permits to attain high gains and to perform detection and localization of ionizing tracks at very high radiation rates. As in other micro-pattern detectors, however, the occasional occurrence of heavily ionizing trails may trigger a local breakdown, with possible harmful consequences on the device itself and on the readout electronics. This paper describes a systematic investigation of the discharge mechanisms in single and multiple GEM structures, and suggests various strategies to reduce both the energy and the probability of the discharges. C 2002 Elsevier Science B.V. All rights reserved.
\end{abstract}

PACS: $29.40 \mathrm{Cs} ; 77.22 . \mathrm{Jp} ; 51.70 .+\mathrm{f}$

Keywords: Gas electron multiplier; GEM; Gas discharges; Streamer; High rate radiation detector

\section{Introduction}

Recently introduced, the gas electron multiplier (GEM) consists of a thin metal-clad polymer foil, perforated with a high density of chemically etched holes, typically ten thousand per square centimeter. On application of a potential difference between the two sides, the foil acts as a charge

*Corresponding author. Tel.: +41-22-7673670; fax: +41-227677100.

E-mail address: fabio.sauli@.cern.ch (F. Sauli). multiplier for electrons released in the gas [1]. Several GEM foils can be used in cascade, increasing the overall gain; a patterned chargecollection anode permits the detection and localization of the primary ionization $[2,3]$.

GEM-based position-sensitive detectors have been developed to operate in high rate, harsh experimental conditions. They are used as charge preamplifiers, in combination with micro-strip gas chambers (MSGCs), to increase the reliability of the HERA-B tracker [4]. A system of multiple GEM detectors with two-dimensional readout is in 
construction for the COMPASS experiment at CERN [5], and similar devices are being developed for the LHCb tracker [6].

The performance of multi-stage detectors exposed to high intensity charged particle beams has been extensively studied in view of their use in present and future high-luminosity accelerators [6-12]. Other high-rate applications under development include medical imaging $[13,14]$ and plasma diagnostics [15].

Using contemporary high-density readout electronics, a proportional gain of several thousand is required for fully efficient detection of minimum ionizing particles in thin layers of gas, typically limited to 2 or $3 \mathrm{~mm}$ in order to minimize time occupancy. This can be attained with a single GEM multiplier followed by a segmented pickup electrode [8].

It has been observed, however, that when operating the detectors at this value of gain, exposure to high radiation fluxes, or the release of a large amount of charge in the sensitive volume may induce a breakdown of the gas rigidity. This is a general feature of most gaseous micro-pattern detectors (MPD), and has been studied at length [2,16-22]. The sequence of events leading to a discharge is initiated when the avalanche size (product of ionization and gain) exceeds a few $10^{7}$ ion-electron pairs, the so-called Raether limit. The ensuing local field modification is then large enough to induce a transition of the avalanche to a forward-backward propagating streamer, a wellstudied process in parallel-plate counters [23] and wire chambers [24,25]. In the latter case, the decreasing field met by the streamer receding from the anode results in termination of the propagation, the limited streamer regime. In MPDs, however, owing to the small distance between electrodes, the streamer is likely to be followed by breakdown. For MSGCs, the discharge problem appeared so serious and damaging as to persuade several groups to abandon the technology [26].

GEM devices have several distinctive advantages over other MPDs. Since the read-out plane is electrically separated from the multiplying electrodes, and as far as a GEM discharge does not propagate all the way to the anodes, the result of breakdown is a large but non-destructive signal.
Moreover, the use of GEM as charge pre-amplifier to another MPD, or several GEMs in cascade, permits to sustain much larger gains than singlestage detectors before breakdown. This feature has been conjectured to be the consequence of a voltage dependence of the discharge limit, since for a given overall gain, each element is operated at reduced potential difference [2]. The GEM electrodes themselves are rather sturdy, and with suitable protection schemes (high value charging resistors and segmentation); they can withstand repeated discharges without damage.

This paper describes the results of a systematic investigation of discharge probability and properties with single and multiple GEM detectors, aimed at increasing their reliability when operated in harsh radiation environments as those met at present and future high luminosity accelerators, or in other applications requiring very high radiation levels.

\section{Experimental setup}

All measurements described here have been performed with small size $\left(10 \times 10 \mathrm{~cm}^{2}\right.$ active area) GEM foils, framed and mounted in versatile detector assemblies described in a previous work [8]. A multi-frame gas containment box permits mounting of one or more GEMs on top of a readout circuit board, and preceded by a drift electrode and a thin window. Distances between electrodes can be varied, making use of insulating spacers bolted on the four corners of the framed electrodes. All GEMs used are of standard design: $50 \mu \mathrm{m}$ thick Kapton with $5 \mu \mathrm{m}$ copper electrodes, and $70 \mu \mathrm{m}$ holes arranged in a triangular pattern with $140 \mu \mathrm{m}$ between centers ${ }^{1}$. For part of the measurements, one of the GEMs was segmented, with one side patterned in four individually powered identical sectors. The separation between sectors, $200 \mu \mathrm{m}$, has been found to introduce no detectable perturbation in the operation, as long as the partition line does not cut through holes.

\footnotetext{
${ }^{1}$ Developed and produced at CERN by A. Gandi and R. De Oliveira, EST-SM.
} 


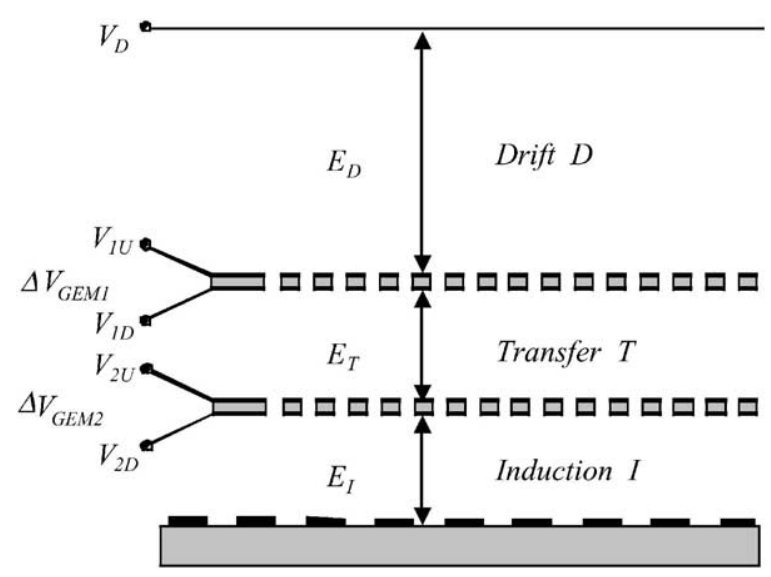

Fig. 1. Schematics of a double-GEM detector.

A double-GEM detector is shown schematically in Fig. 1; conventional notations for the different elements are indicated. For single or triple GEM, obvious modifications to the notations apply. For signal readout, we have used a board with parallel anode strips, $150 \mu \mathrm{m}$ wide at $200 \mu \mathrm{m}$ pitch, connected in group by wire bonding or conductive epoxy to an external grounding circuit. Unless otherwise mentioned, the induction and transfer gaps were $2 \mathrm{~mm}$ thick, and the drift gap $3 \mathrm{~mm}$. Most measurements have been performed in a non-flammable argon-carbon dioxide mixture in the volume proportions 70-30, a choice optimized for its performance and resilience to aging [27]. In Section 6, results obtained with other mixtures are described.

Whilst not affecting the occurrence of the initial discharge, the high voltage powering scheme can dramatically alter the sequence of ensuing events. As mentioned in a previous work [28], use of individual units to power each electrode (through protection resistors), convenient for systematic studies, can result in a sudden increase of the potential difference across a GEM electrode leading to sustained discharges, often with irreversible damages to the foil. This can be imputed to the operating characteristics of most HV units, not designed to drag reverse current, and therefore reacting erratically to overloads. When using a safer resistor chain network for powering the various elements, results are more predictable; the

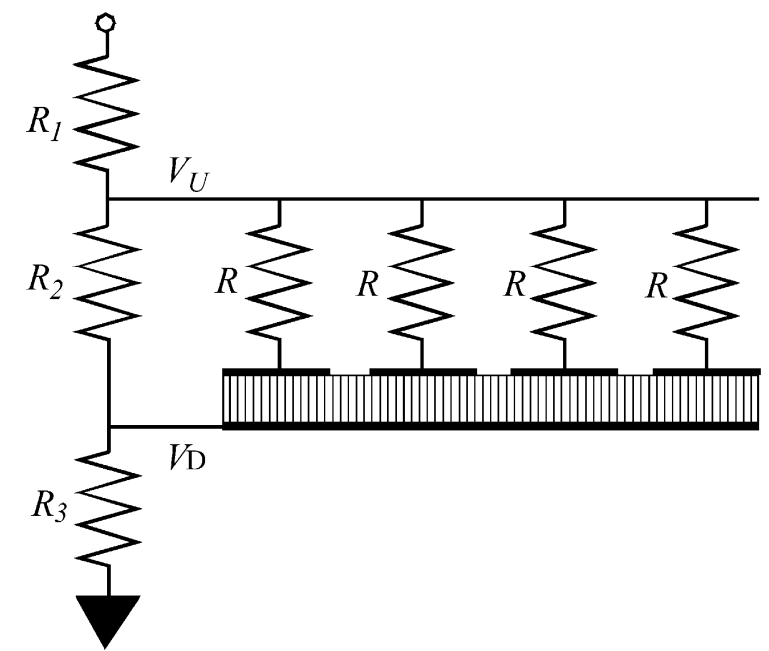

Fig. 2. Resistor partition network used to power a sectored GEM.

propagation probability, however, appears to be affected by the value of resistors. Indeed, as we will discuss later, the sudden drop of the potential difference between electrodes interested by a discharge increases the external fields, and as a consequence the propagation probability between GEMs and from a GEM to the read-out board. Fig. 2 shows, schematically, a resistive network used for powering a segmented GEM; values of the resistors in the main chain are selected to obtain the appropriate voltages. The individual protection resistors $\mathrm{R}$ are chosen to ensure that, in case of a discharge, most of the potential drop occurs on the upper side of the sector. Whilst obviously the best protection is obtained with very high value resistors, the experimental requirements (particle rate and gain) determine the maximum values that can be used in order to maintain the potential drops under beam load within acceptable limits. Typically, we have used values of $0.5-1 \mathrm{M} \Omega$ for the main chain $\left(R_{1}, R_{2}\right.$ and $\left.R_{3}\right)$ and 10-20 M $\Omega$ for the protection resistors on sectors. At an integral particle rate of $10^{8} \mathrm{~s}-1$ over the sensitive area, the maximum expected in the COMPASS experiment, this implies, at a nominal gain of $5 \times 10^{3}$, an overall detector current of $\sim 1 \mu \mathrm{A}$, with a resulting potential drop of $5 \mathrm{~V}$ on each multiplier. A characteristic of this powering scheme is 
that, in case of an accidental permanent short in one sector, the potential in all remaining sectors of the affected GEM drops slightly, improving the overall safety of operation. If needed, a small increase in the applied voltage can then be used to restore the gain of unaffected sectors, at the cost of a moderate increase in the current.

The appearance of a discharge is recognized electronically as a fast and exceedingly large pulse on the concerned electrodes, with a charge roughly corresponding to the product of voltage and source capacitance. Large induced signals are also seen in all other electrodes. In most cases, a discharge is detected also as an overload of the power supply, usually set to a limiting value of maximum current. At the beginning of this study, discharges were monitored making use of capacitive dividers on various electrodes; it was found, however, that the external circuit could substantially modify the discharge energy and voltage recovery, as well as induce interference between signals. The scheme was abandoned in favor of resistive dividers, typically several hundred $M \Omega$ in series with the $50 \Omega$ load on the oscilloscope. For the most relevant part of the study, concentrating on the discharge propagation to the (grounded) read-out electrode, signals were detected directly on the properly terminated anode board.

Fig. 3 shows, for a single GEM detector, an example of the sequence of discharge events, recorded with a four-track digital oscilloscope.

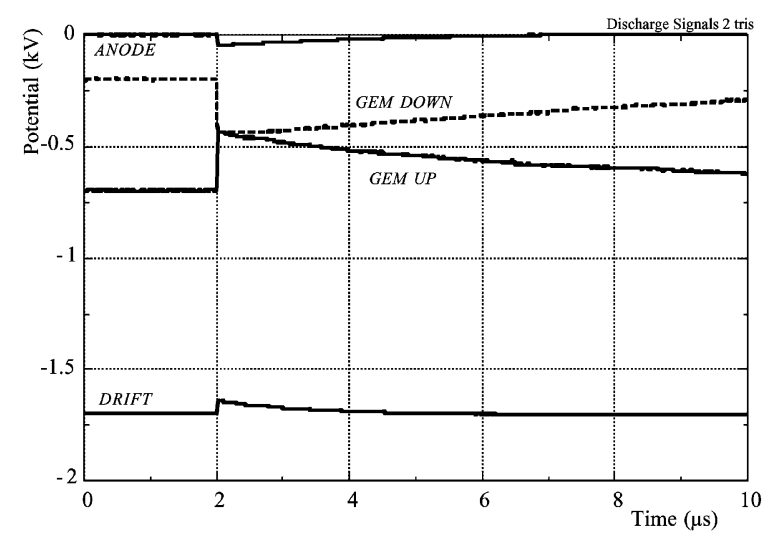

Fig. 3. Non-propagating discharge in a single GEM detector: voltage levels and signals on all electrodes.
As a function of time, the vertical scale represents the voltage on electrodes: top to bottom, the anode strips, the GEM side facing the anodes, the opposite side of the same and the drift electrode. In this particular event, the discharge affected only the multiplier: the voltage difference across GEM drops symmetrically to zero (no protection resistor $\mathrm{R}$ was used in this case). A fraction of the electrons generated in the discharge is collected at the anodes, providing a negative pulse, and a positive induced signal is seen on the drift electrode. The recovery time of the pulses in the plots (around $10 \mu \mathrm{s})$ is determined by the circuit used for readout, a $10 \mathrm{nF}$ capacitor on $50 \Omega$, and does not correspond to the real response of the HV circuit (several ms).

To induce controlled discharges, two methods were employed to generate the large ionization densities emulating realistic operating conditions. In both cases, the discharge probability is defined as the ratio between the observed frequency of breakdowns and the source rate.

An internal gaseous alpha particles emitter, ${ }^{220} \mathrm{Rn}(6.4 \mathrm{MeV})$, added to the gas flow. The method has the advantage of uniformly exposing the sensitive volume of the detector; in a thin $(3 \mathrm{~mm})$ drift gap; the energy loss distribution has a broad spectrum with an average around $400 \mathrm{keV}$ [29]. With the natural thorium oxide radon generator used, counting rates are, however, small, typically a few $\mathrm{Hz}$. With a reasonable waiting time of a few hours for the occurrence of a discharge, this sets a practical limit to measurable probabilities of around $10^{-4}$.

A collimated ${ }^{241} \mathrm{Am}$ radioactive source, emitting $5.6 \mathrm{MeV}$ alphas, is mounted either internally, in contact with the drift electrode, or externally through a thin $(3.5 \mu \mathrm{m})$ polymer window. In both cases, the drift electrode is made of a fine wire mesh, to allow penetration of the radiation into the drift volume. In this case, the energy loss spectrum is better defined, with a peak around $500 \mathrm{keV}$; rates of several hundred $\mathrm{Hz}$ can be achieved with a coarsely collimated source, permitting to measure in reasonable time discharge probabilities down to $6 \times 10^{-6}$. Though more flexible, in particular because it allows changing the angle of the tracks, the external mounting has 
the inconvenience of exposing small areas of the detector at a time, and to make it less stable in operation, probably due to water permeation into the gas volume.

It should be emphasized that the probability of the transition from proportional multiplication to discharge at a given voltage depends on many internal and external factors, such as temperature, humidity and gas flow, as well as on quality and previous history of the electrodes. Whilst shortand medium-term comparisons varying a single parameter can be considered significant, caution should be used when confronting measurements realized in altered situations or with different detectors.

A condition found to radically tamper the results is the accidental loss of connection to ground on some readout strips. In our setup, this could accidentally happen due to the fragility of the wire bonds between anodes and terminating board. When irradiating a partly damaged detector, probably due to the floating strips charging-up until the occurrence of micro-discharges, a correlation was seen between discharge and signal rates in suspicious regions (see Fig. 4). Due to various manipulations between the two measurements, the two plots do not correlate fully, but are indicative of the trend. Another frequent source of problems is the occurrence of external breakdowns in cables or connections. The long intervals between dis-

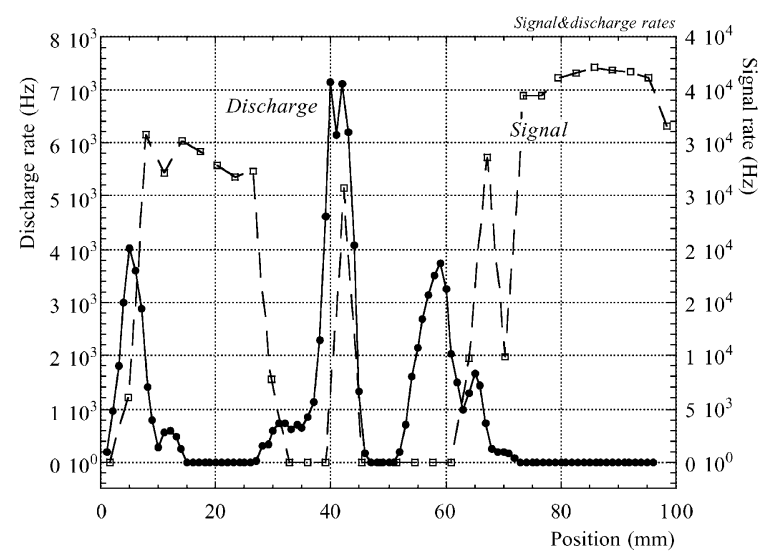

Fig. 4. Discharge and detected source rate as a function of position. charges, several hours, can obviously distort the results.

The study of discharge rates with alpha particles provides only qualitative indication of the behavior of the detector in real running conditions, because of the different spectrum and angular distribution of heavily ionizing tracks in a real experiment. A definitive answer concerning the detector performance has necessarily to be obtained on the experiment.

\section{Gain measurement and calibration}

An accurate estimate of gain in each operating condition is essential for proper interpretation of the results. To this end, the detector was mounted in front of an X-ray generator; the absolute gains, and the charge sharing ratios between electrodes, are then estimated from the measured current $I$ at a known value of flux $R$ through the expression,

$M=I(e N R)^{-1}$

where $e N$ is the ionization charge produced in each conversion.

During long-term exposures to highly ionizing radiation, the gain could be regularly checked recording the pulse height of the $5.9 \mathrm{keV}$ line from a ${ }^{55} \mathrm{Fe}$ X-ray source. All measurements of gain in this paper refer to the "effective" gain, i.e. the amount of electron charge collected on the readout electrode divided by the primary charge. Depending on operating conditions, the total amount of charge flowing through the detector can be larger than the fraction being collected by the various electrodes.

Detailed studies of gain and charge sharing as a function of drift, transfer and induction field have been published [28]. We reproduce here some data relevant to the present study. Fig. 5 provides the gain dependence on voltage for a single, double and triple detector, measured with soft X-rays. On multiple devices, voltages across GEMs were kept identical, with transfer and induction fields of $3.5 \mathrm{kV} \mathrm{cm}^{-1}$. In view of the dependence of effective gain on the value of external fields, this has to be considered only as a representative example; in the course of the measurements the 


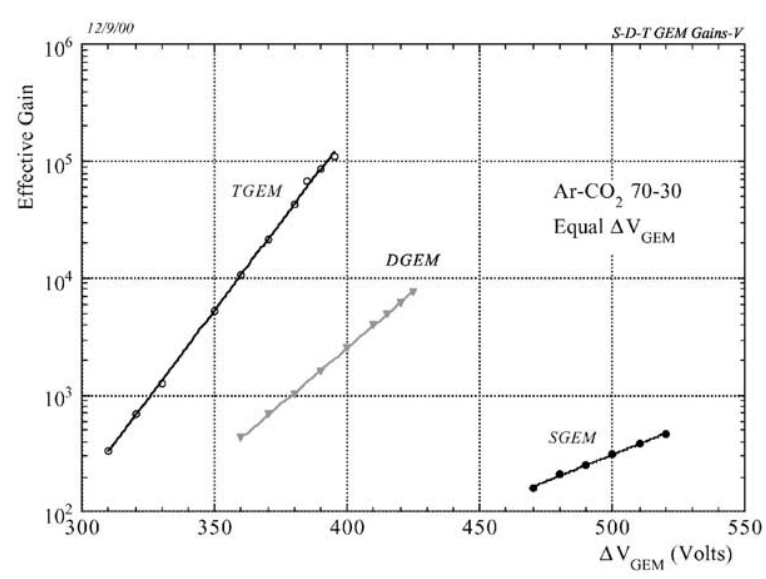

Fig. 5. Total effective gain on anodes for a single, double and triple GEM detector.

actual value of gain was continuously monitored using the method described above.

\section{Discharge in single and multiple gem structures}

The sequence of events that can lead to the propagation of a discharge throughout the detector begins with a sudden, radiation-induced breakdown of the gas rigidity in one GEM, normally the last in a cascade of multipliers. The probability of this initial discharge appears to depend on the primary ionization density and on the overall gain of the structure, but not directly on the external fields. Reproduced from an earlier work, Fig. 6 provides the discharge probability as a function of voltage for a single GEM detector, observed with the internal ${ }^{220} \mathrm{Rn}$ alpha source added into the gas flow [2]. The measurement suffers from the statistical limitations discussed in Section 2, but has the particularity, as compared to most of the following observations, of corresponding to an isotropic track distribution in the narrow gap, and therefore to a large range of ionization density. The threshold for discharges is observed at a gain of around one thousand.

A better defined energy loss can be obtained with an alpha source directly placed over the semitransparent drift electrode, and releasing $\sim 2 \times 10^{4}$ ionization electrons per track in the drift gap at a

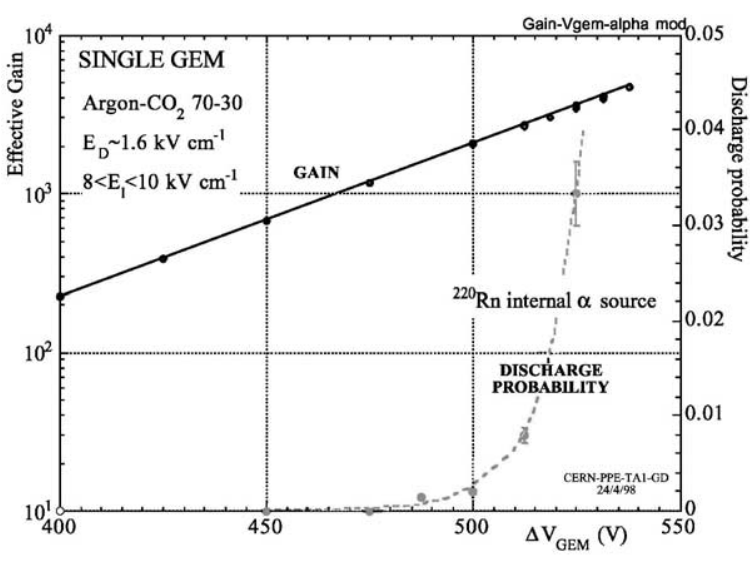

Fig. 6. Discharge probability with the ${ }^{220} \mathrm{Rn}$ internal alpha source and gain as a function of voltage for a single GEM detector.

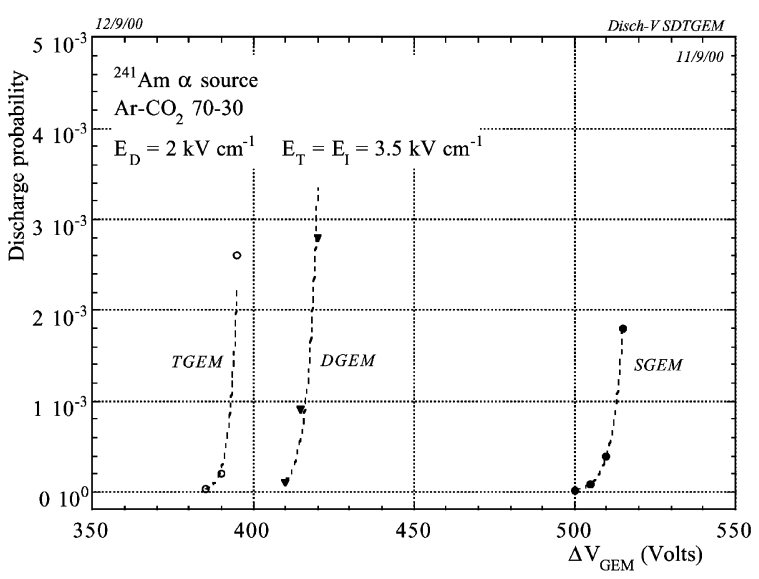

Fig. 7. Discharge probability on alphas in single, double and triple GEM detectors, as a function of applied voltage (equal on all GEMs).

rate close to $100 \mathrm{~Hz}$. This charge is drifted into the first GEM, undergoes a process of multiplication and, for multiple structures, is transported into and further amplified by the cascaded GEMs. Fig. 7 shows the discharge probability measured as a function of multiplying voltage, in the single, double and triple GEM, exposed to the alpha source. For this measurement, the voltage applied to each multiplier was identical (equal gain sharing); induction and transfer fields were fixed at $3.5 \mathrm{kV} \mathrm{cm}^{-1}$, and the drift field at $2 \mathrm{kV} \mathrm{cm}^{-1}$. 


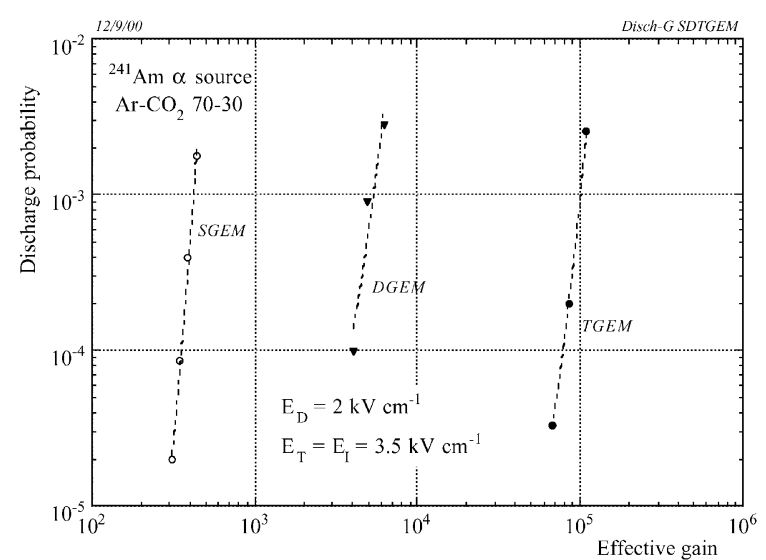

Fig. 8. Discharge probability as a function of total effective gain for single, double and triple GEM detectors.

The decrease in discharge voltage is, of course, a reflection of the increasing avalanche size for multiple devices. In Fig. 8, the same data are plotted as a function of effective total gain of the structures. In the condition described, the maximum gain is increased by around an order of magnitude at each addition of a multiplier. In both measurements, as well as in the following ones, the statistical significance of the zero baseline corresponds to the observation of no discharges during a waiting time of $3000 \mathrm{~s}$, or a probability of less than $6 \times 10^{-6}$.

The discharge probability depends strongly on the angle of tracks, as shown in Fig. 9 for a single GEM. For this measurement, the collimated ${ }^{241} \mathrm{Am}$ source was placed outside the detector, entering the sensitive volume through a thin $(3.5 \mu \mathrm{m})$ mylar window. The angular opening of the beam was about $\pm 30^{\circ}$ around the axis, and the measurement was done with the axis perpendicular to the detector, or inclined by $\sim 45^{\circ}$.

As shown in a previous work, maximum gain and discharge probability depend on the charge sharing between cascaded multipliers [2]. For the double detector, Fig. 10 shows combined gain curves obtained by varying the voltage on each GEM; the dashed line represents the natural discharge boundary, without radiation. An optimum is reached when the first multiplier has a voltage $5-10 \%$ larger than the second; with

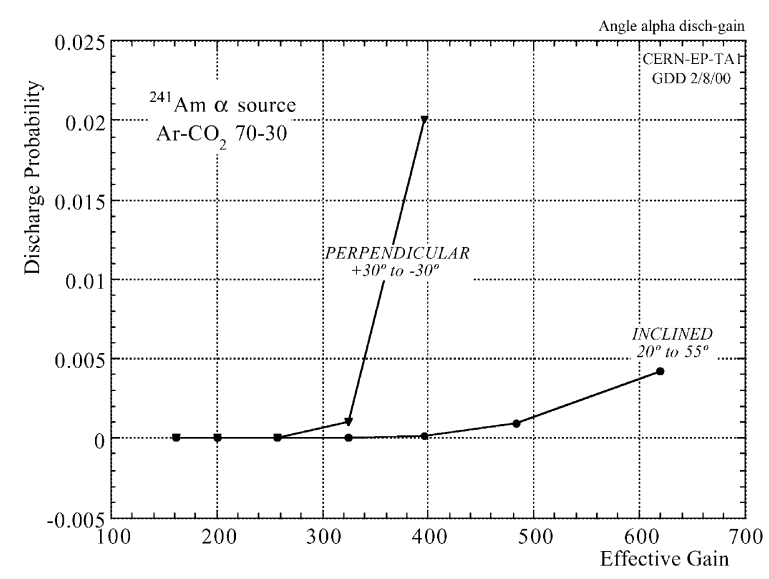

Fig. 9. Dependence of discharge probability for the single GEM for two values of the alpha particle average incidence angle.

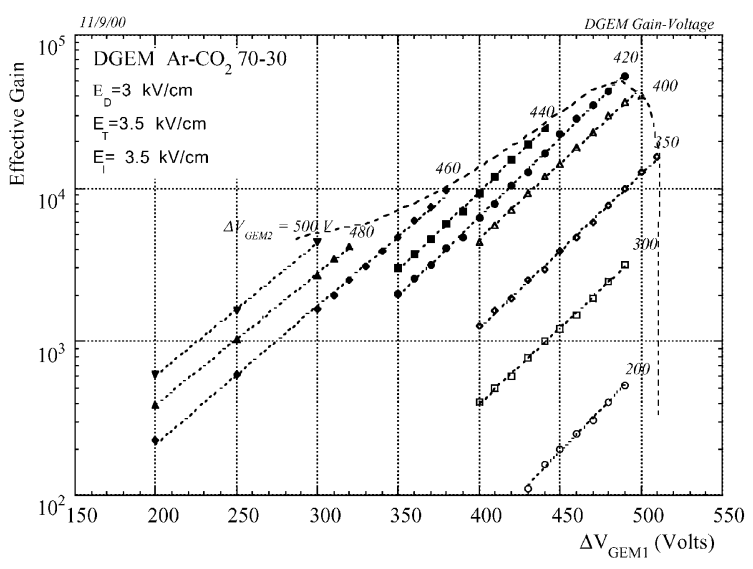

Fig. 10. Combined gain curves for the double GEM detector as a function of voltage on the two multipliers. The dashed curve represents the maximum gain boundary at low irradiation levels.

reference to Fig. 5, this corresponds to a gain of GEM1 exceeding the gain of GEM 2 by $50-100 \%$. Possible explanations for this behavior are discussed in Section 7. Fig. 11 provides the discharge probability, under alpha irradiation, as a function of the relative voltage offset between the two GEMs, their sum (and therefore the total gain) being constant for each curve, and for three values of total effective gain $G$. In the figure, the 


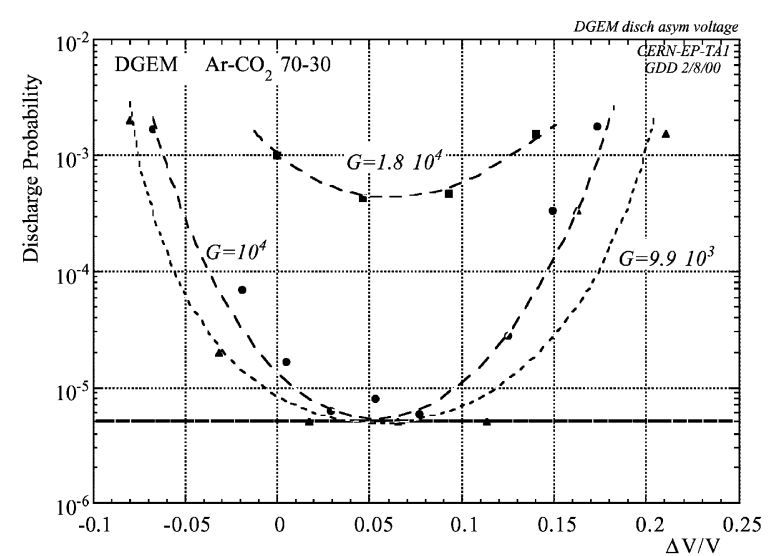

Fig. 11. Discharge probability on ${ }^{241} \mathrm{Am}$ alphas for the double GEM, as a function of the voltage imbalance, and for three values of total gain.

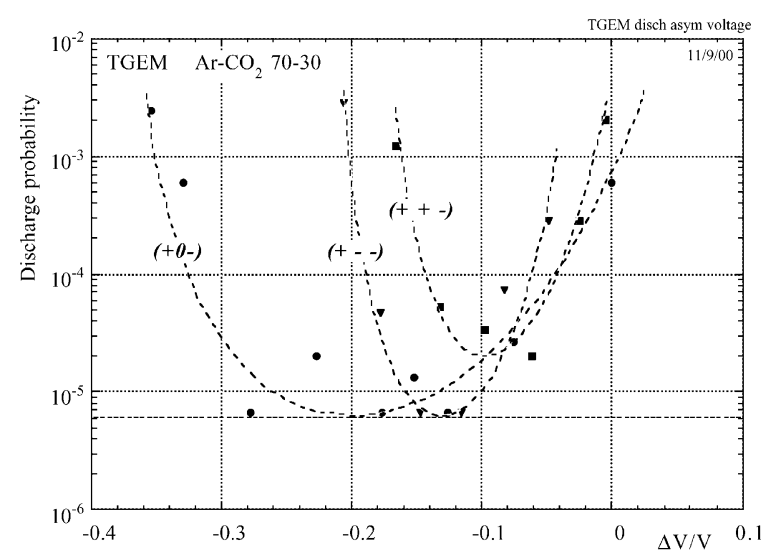

Fig. 12. Discharge probability for the triple GEM as a function of the asymmetry in applied voltages (for the meaning of symbols, see text).

horizontal dotted line represents the statistical limit to the measurements.

A similar observation is made for the triple GEM detector, Fig. 12. As a function of the percentage difference in operating voltages, the discharge probability is measured for three types of offset: equal voltage on GEM1 and GEM2, and lower on GEM3 (indicated as ++- ); equal and lower voltage on GEM2 and GEM3 $(+--$ ) and symmetric offset on GEM1 and GEM3 $(+0-)$. In the figure, the horizontal scale is the voltage difference between GEM1 and GEM3, divided by the voltage on GEM2. In all cases, the overall gain is kept constant ensuring an equal sum of operating voltages. The best choice seems to be the asymmetric condition $(+0-)$, with GEM1 (respectively, GEM3) higher (lower) in voltage by $\sim 10 \%$ as compared to GEM2. Albeit moderate, the observed improvement in discharge rate can be exploited in the design of an optimized detector. The fast increase on both sides of the minimum suggests great caution when comparing results obtained with different detectors and powering schemes.

It is unknown at this point if the geometry of GEMs, and in particular the diameter of holes, plays a role in defining the optimum. It has been observed in the early developments that the maximum reachable gain increases when reducing the diameters of the holes [27]. Since, given the input charge, higher gains can be sustained when the multipliers operate at lower voltages (see the discussion section), it might be inferred that narrowing the holes for the last multipliers in a cascade should be advantageous. A competing mechanism, the reduction of electron transparency when narrowing the holes at fixed values of external field, may invalidate the previous argument. This point certainly requires further investigation.

\section{Localized and propagating discharges}

As discussed previously, although the first rupture of gas rigidity occurs in the high field of a GEM, normally the last in a cascade, the discharge can propagate forward and backwards to other electrodes. A fast propagation between GEMs has been observed, compatible with a photon-mediated breeding of charge by ionization of the gas or of the metal electrodes [30]. In the present study, motivated more by the requirement to reduce the discharge occurrence than to analyze their formation mechanism, we have made no distinction based on propagation time. However, the predominance of a fast propagation mechanism between GEMs is confirmed by the observation that discharges can propagate between two multipliers, even if the electric field is inverted in 


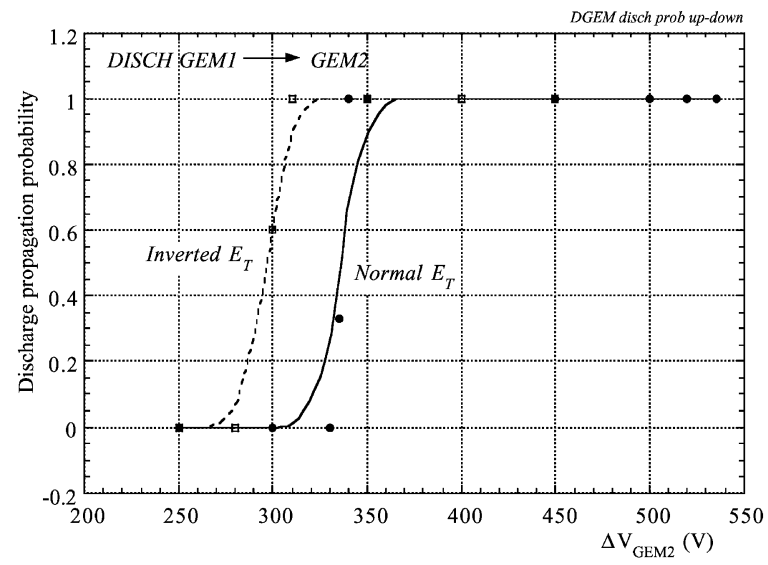

Fig. 13. Discharge propagation probability between first and second GEM in a cascade, as a function of voltage on the second, for normal and inverted transfer fields.

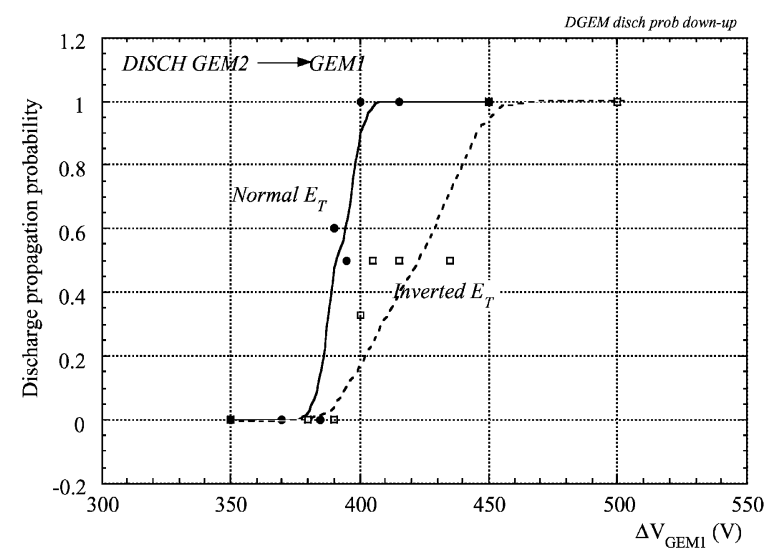

Fig. 14. Discharge propagation probability between second and first GEM in a cascade, as a function of voltage on the first, for normal and inverted transfer fields.

the transfer region. Figs. 13 and 14 provide the measured discharge propagation probability, from GEM1 to GEM2, and, respectively from GEM2 to GEM1, as a function of the voltage on the other multiplier, for two values of the transfer field, normal and inverted. In the figures, as well as in the following, the discharge propagation probability is defined as the ratio of the observed propagating to the total GEM discharges. To avoid damages to the electrodes, the potential on the first discharging GEM was set just above the onset of the discharge probability, see Fig. 6; possible effects of this potential on propagation probabilities were not investigated.

An examination of the electric field map in the double detector with inverted transfer field reveals an enhancement of the field strength on the metal rims around the holes on the more negative (cathode) side of the second multiplier. Photons emitted by a discharge in GEM1 can therefore release photoelectrons in the anomalous high field on the upper side of GEM2; they will then be preamplified before entering the normal multiplication region in the holes. The large gain enhancement may explain the lower threshold for propagation for this configuration (Fig. 13). The process is not symmetric, as the field on the cathode of GEM1 is not increased by the transfer field inversion. Moreover, photons emitted by a discharge in GEM2 have to traverse the holes in GEM1 and ionize the gas to produce any effect, possibly explaining the higher discharge threshold in the inverted configuration (Fig. 14). This point certainly requires further investigation.

The forward propagation to the anodes, and backwards to the drift electrode appear instead to be slower processes, involving electron and ion transport. In the present work, we have concentrated on the study of conditions leading to the propagation of a discharge from the last GEM to the anode, the most dangerous one, since it can result in permanent damage of the read-out electronics. It should be stressed that all along our tests, as far as basic constraints on discharge energy are observed and safe power supply schemes used, no physical damage resulted to the electrodes after hundreds of localized discharges.

The energy in a primary GEM discharge depends on the capacitance between electrodes, and increases with their surface. Making use of the segmented $10 \times 10 \mathrm{~cm}^{2}$ GEM described in Section 2 , we could vary this capacitance in discrete steps between 1.4 (one sector) and $5.6 \mathrm{nF}$ (full area). In order to expose the entire area of the detector, this measurement was made using the internal ${ }^{220} \mathrm{Rn}$ source. For non-propagating discharges, a clear correlation is seen between the (negative) charge collected on the anodes and the available energy, 


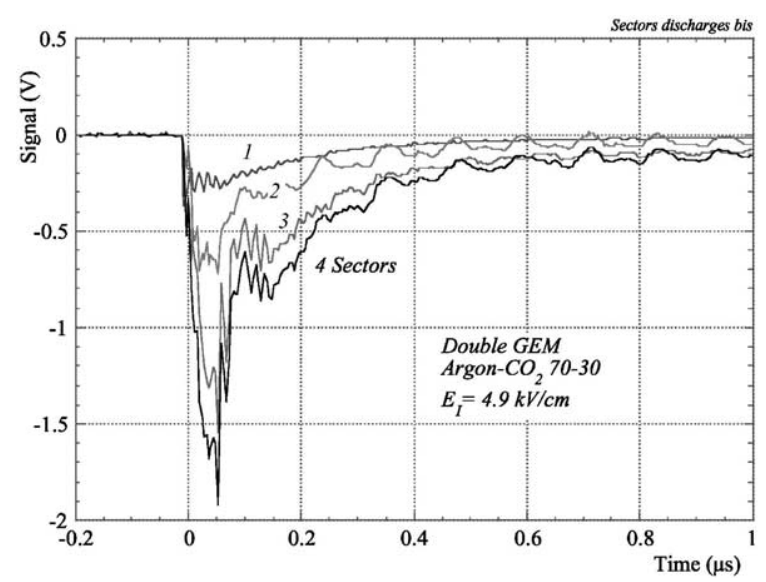

Fig. 15. Discharge signals on anodes for increasing GEM capacitance, obtained by grouping one to four sectors.

as seen in Fig. 15. For this measurement, and to limit the signal induction due to capacitive couplings, small groups of strips in the readout board were connected to the recording oscilloscope, properly terminated. In the case of a standard non-sectored $10 \times 10 \mathrm{~cm}^{2}$ GEM, the detected pulse has an amplitude of around $2 \mathrm{~V}$, and corresponds to about $4 \mathrm{nC}$ of collected charge, generally considered harmless for the electronics.

The flow of charge is larger by more than an order of magnitude in the case of a fully propagating spark, see Fig. 16. The released energy in this case is determined by the capacitance between the read-out board and the lower GEM electrode, around $50 \mathrm{pF}$, and the operating voltage $(1.6 \mathrm{kV})$; it corresponds to about $50 \mathrm{nC}$.

The probability for a GEM discharge to evolve into a full discharge to the anodes depends both on the induction field strength and on the energy of the primary discharge, as shown in Fig. 17 that provides the fraction of propagating discharges. The four dashed lines labeled "Sectors Up" are an eye fit through measurements made on a detector having the sectored GEM (the second of two in cascade) with the partitions towards the drift electrode. A propagating discharge in this case takes place between the anode plane and the full lower GEM electrode. Thanks to the power supply scheme used for segmented electrodes, with individual protection resistors (see Section 2), a

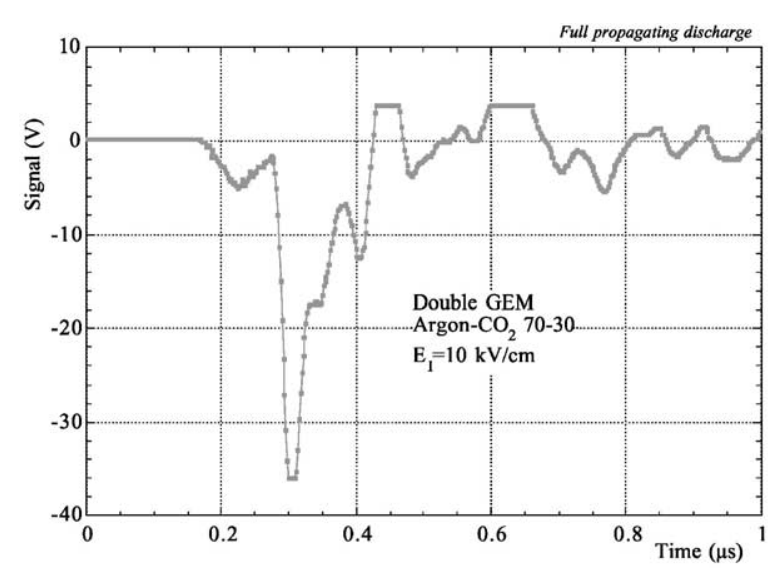

Fig. 16. Anode signal for a fully propagating discharge.

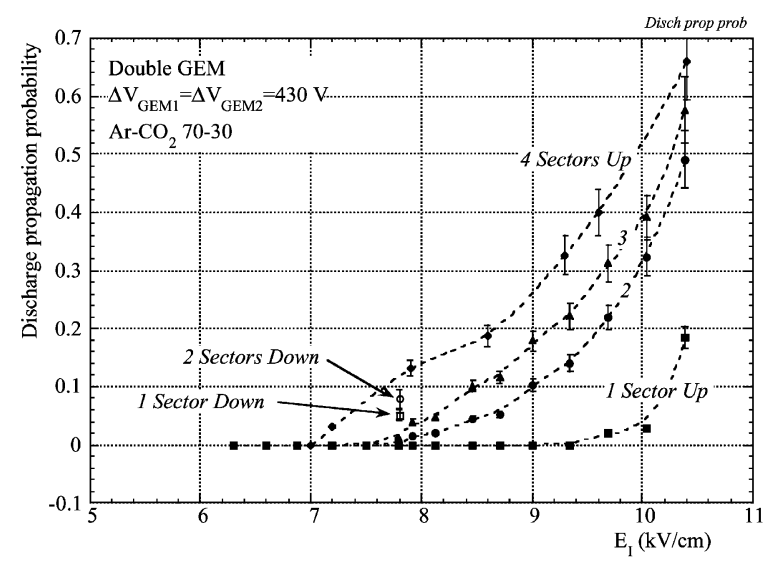

Fig. 17. Discharge propagation probability as a function of induction field for a sectored GEM.

discharge in the multiplier results only in a small $(10 \%)$ increase of the field in the induction gap. The upper, or transfer field, is of course locally increased, but this does not affect the downward propagation probability.

The two unconnected points (open symbols) provide instead the propagating discharge probability measured inverting the last GEM, with the four sectors facing the anodes; the noticeable raise in discharge probability is consistent with the sudden increase of the induction field during the discharge due to the resistor protection scheme. This suggests the addition, whenever possible, of a 


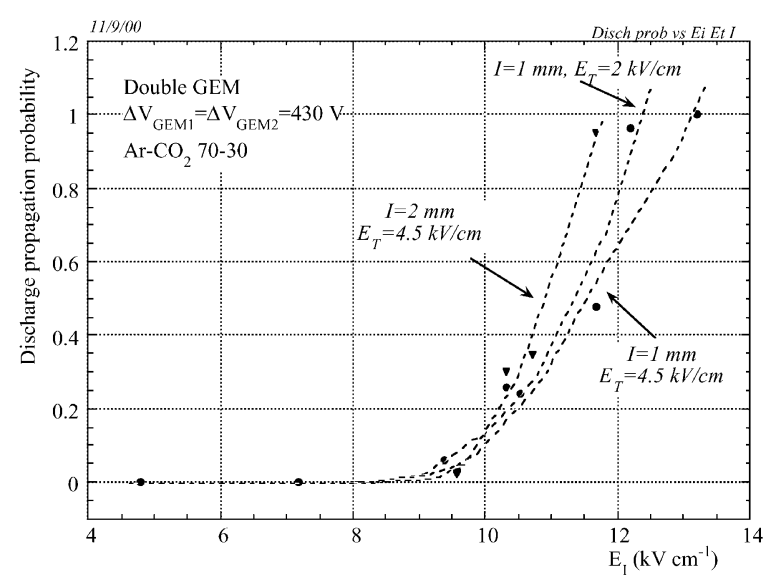

Fig. 18. Discharge propagation probability for several values of the induction gap $I$ and transfer field $E_{\mathrm{T}}$.

protection resistor on the GEM side opposite to the induction gap also in the case of nonpartitioned foils. In order to profit from the decrease both in discharge energy and probability, one could envisage partitioning the multiplier on both sides. Powering the sectors through individual protection resistors with suitably chosen values, one could limit the discharge energy and at the same time ensure that most of the potential drop occurs on the side opposite to the induction gap. This scheme, however, complicates the power distribution, and has not been tried yet.

The full propagation probability is almost independent of the thickness of the induction gap and the strength of the transfer field between the two GEMs, as shown in Fig. 18. On the contrary, the addition of an external capacitor in parallel to the induction gap, itself having around $50 \mathrm{pF}$, appears to lower the threshold considerably for propagating discharges (Fig. 19). The reasons for this behavior have not been understood, and suggest great caution in interpreting the results, particularly if external components are added to filter noise or to read-out pulses from electrodes.

From the described results it can be inferred that the full discharge propagation has a non-zero probability only at values of induction field above a threshold value around $5-6 \mathrm{kV} \mathrm{cm}^{-1}$. We have chosen a value of $3.5 \mathrm{kV} \mathrm{cm}^{-1}$ as a standard safe operating point, although this implies a moderate

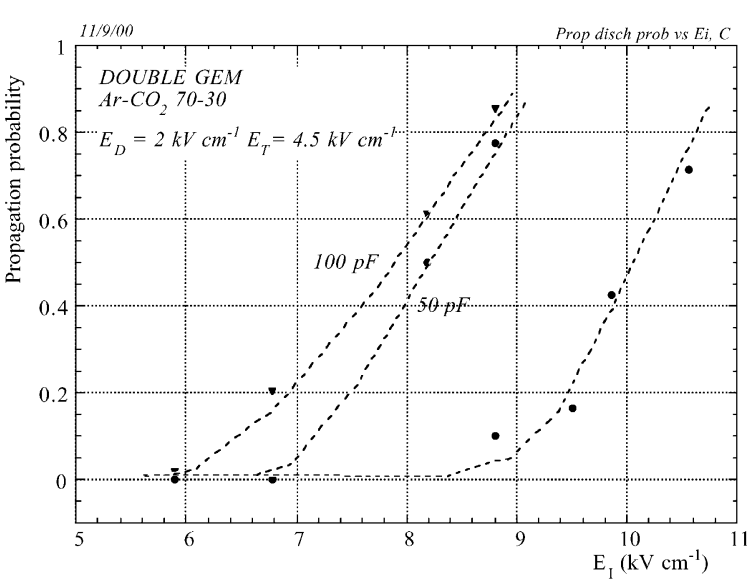

Fig. 19. Discharge propagation probability without (rightmost curve) and with additional capacitors in parallel with the induction gap.

loss in the electron transfer ratio (see for example Ref. [28]).

\section{Influence of gas filling and transfer gap}

All measurements described so far have been made with a 70-30 mixture of argon and carbon dioxide, a convenient choice for detectors to be used in harsh radiation environments because of non-flammability, large drift velocity, low diffusion, non-polymerizing properties and low cost. It has, however, been observed already in earlier works that other gas mixtures permit reaching higher gains, at least in absence of heavily ionizing radiation [31]. We have investigated systematically gain and discharge limits in mixtures of argon and neon with $\mathrm{CO}_{2}$ and $i-\mathrm{C}_{4} \mathrm{H}_{10}$; only the most significant results are reported here.

Figs. 20-22 show the effective gain of single, double and triple GEM structures in various gases. Gains were measured exposing the detectors to a low intensity $5.9 \mathrm{keV}$ X-ray source, and deduced from the detected charge using the cross-calibration method described in Section 2. For all measurements shown, the drift field and gap are $2 \mathrm{kV} \mathrm{cm}^{-1}$ and $3 \mathrm{~mm}$, whilst transfer and induction fields are $3.5 \mathrm{kV} \mathrm{cm}^{-1}$ with $1 \mathrm{~mm}$ gaps. Although measurements were taken for other 


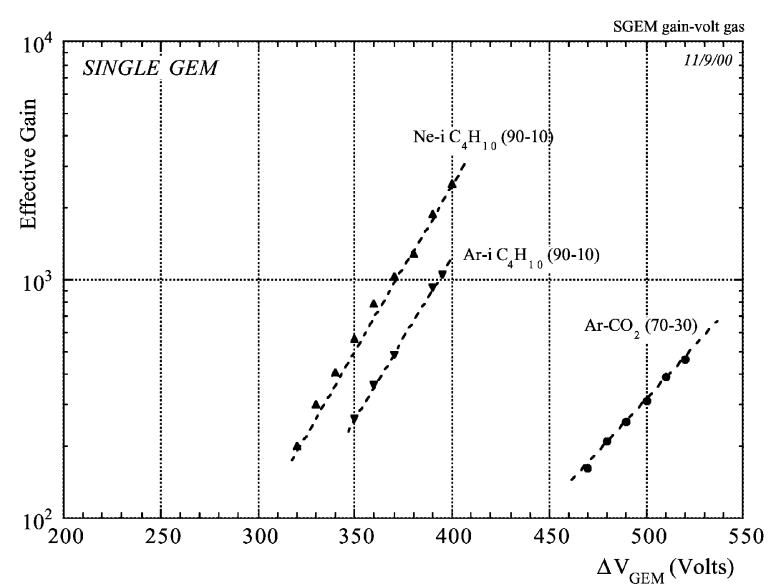

Fig. 20. Effective gain of a single GEM detector in various gas mixtures.

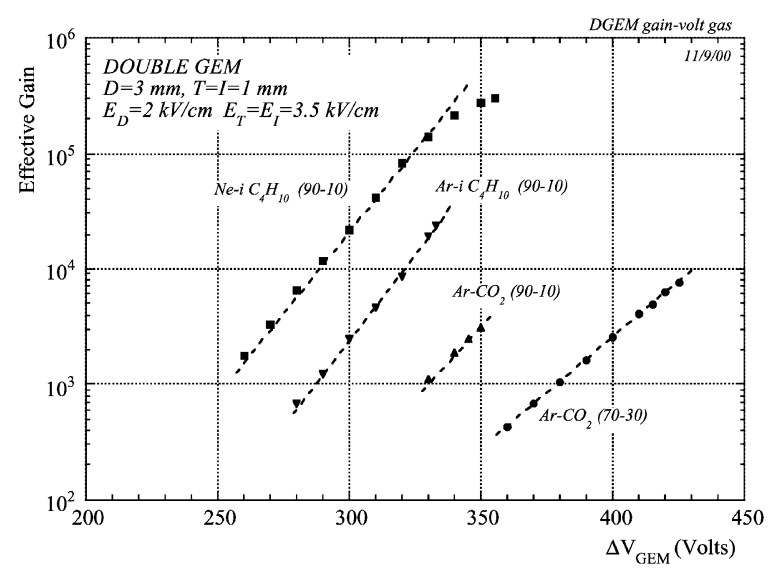

Fig. 21. Effective gain of a double GEM detector in various gas mixtures.

values of gaps and external fields, the dependence of gain on these parameters in the range covered is weak.

Exposing the detector to an external collimated alpha source, we have measured the discharge probability in the various gas mixtures as a function of gain for several values of transfer and induction gaps, Figs. 23 and 24. From the data, and by comparison to Figs. 7 and 8, one can deduce a general trend for the detectors to withstand larger gains in relatively poorly quenched mixtures with isobutane; particularly

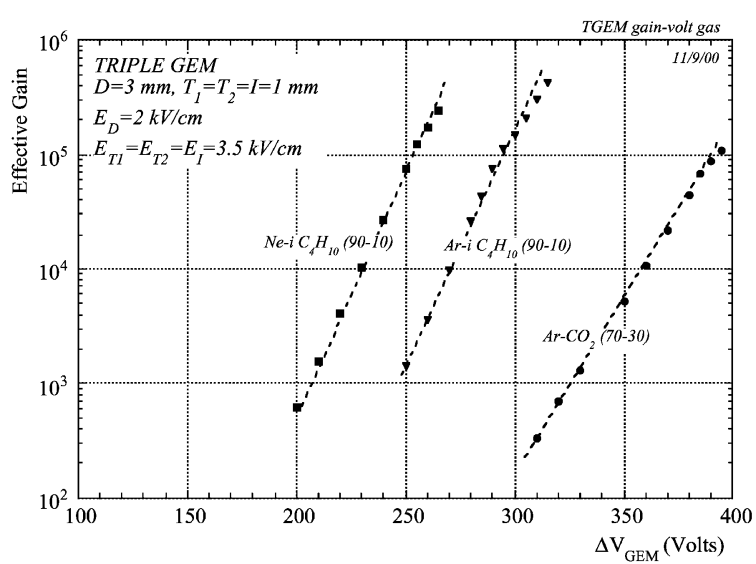

Fig. 22. Effective gain of a triple GEM detector in various gas mixtures.

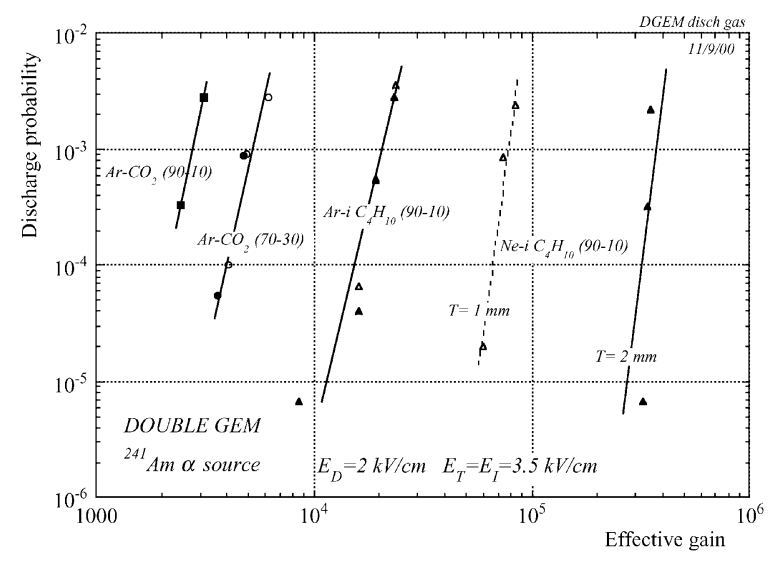

Fig. 23. Double GEM discharge probability under alphaparticle irradiation, as a function of gain, in various gases. Open markers correspond to a transfer gap $T=1 \mathrm{~mm}$, full markers to $T=2 \mathrm{~mm}$.

large gains are attained in neon. Disadvantages of such a choice will be discussed in the next section. A strong dependence of maximum gain at discharge on the thickness $T$ of the transfer gap is observed for the double structure in neonisobutane, Fig. 23. For argon mixtures, on the contrary, data points for transfer gaps of 1 and $2 \mathrm{~mm}$ overlap. One can speculate on the presence of strong photon feedback mechanisms in neon mixtures between the two GEMs, more effective 


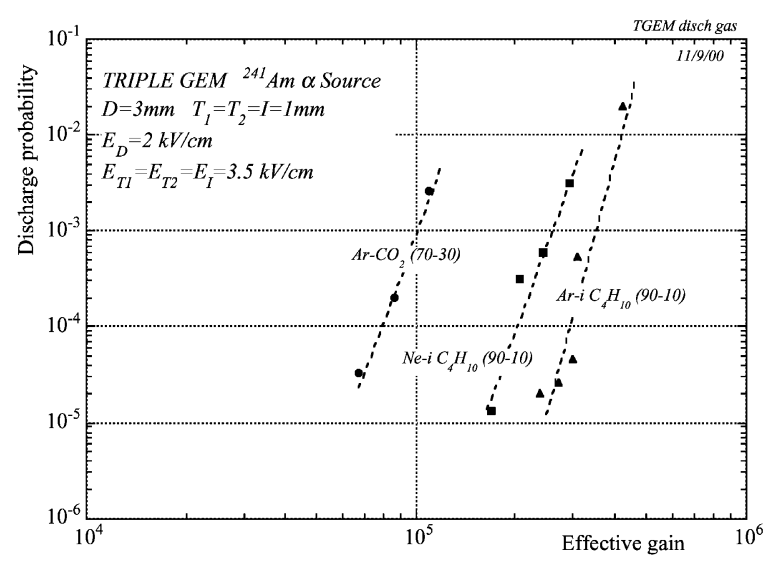

Fig. 24. Triple GEM discharge probability under alpha-particle irradiation, as a function of effective gain, in various gases.

for narrow gaps because of reduced gas selfabsorption. Measurements in the triple GEM (Fig. 22) were taken only with $1 \mathrm{~mm}$ transfer gaps, but presumably the same trend would appear using wider spacing. The inconvenience of such a choice is a larger avalanche size, increased by diffusion, and the need to operate the detector at very high voltages.

\section{Discussion of the results}

Some general features emerge from the described measurements. In presence of moderate irradiation, proportional gains of several thousand can be reached with a single GEM gains well above $10^{4}$, and, respectively $10^{5}$ can be attained cascading two or three multipliers. The maximum gain that can be safely held is, however, strongly reduced when the detectors are exposed to heavily ionizing particles. Given the gain, the discharge probability depends both on the released charge amount and density and on the space distribution of the ionization trail (angle of tracks). With multiple structures, the safe gain increases again by about an order of magnitude at each addition; the discharge probability is further reduced offsetting the voltages in order to enhance the gain in the first element in the cascade.

Two possibly concurrent explanations can be given for the observed behavior. On one hand, mounting several devices in cascade results in an increase of the size of the charge cloud propagating through the detector, and therefore a decrease of its density, due to electron diffusion. On the other hand, the same value of gain is reached in multiple devices and in some mixtures at substantially lower voltages applied across each element, as compared to a single multiplier operated in $\mathrm{CO}_{2}$-rich mixtures. A diffusion-dominated density dependence of the breakdown voltage has been proposed by the authors of Ref. [16]. Alternatively, we favor the hypothesis of a voltage dependence of the Raether limit, with the structures being capable of holding more charge when operated at lower voltage. Indeed, it is rather intuitive that, in order to locally reach the field strength necessary for the transition, a larger charge density is required in presence of a lower field. At present, the experimental data do not seem to permit disentangling between the two possibilities. One should, however, consider that the increase in the charge cloud size going from single to multiple devices is rather modest, in view of the short distance between the elements, $2 \mathrm{~mm}$ or less (the electron diffusion increases only with the square root of the drift length). Moreover, very little dependence, if any, of the discharge point on the drift field has been observed, in a range of fields that would change the electron diffusion by more than an order of magnitude.

An increase of the charge spread, due to geometry or diffusion, could negatively affect the detector performance, reducing both localization accuracy and two-track resolution. The electron drift velocity is a factor of two lower in the isobutane mixtures, as compared to argon-carbon dioxide, worsening both time resolution and high rate performance. Moreover, as seen for example in Fig. 20, the gain-voltage characteristics is steeper in the poorly quenched mixtures, raising concerns about the general mechanical tolerances required for a uniform performance of large size detectors. The isobutane mixtures are also flammable, and presumably much more susceptible to aging at high radiation levels.

A second observation having important practical consequences is that not only the discharge energy, but also the propagation probability 
throughout the detector depends on the capacitance of the GEM. Manufacturing the multipliers with independently powered sectors therefore largely reduces the probability of energetic discharges propagating into the delicate read-out electronics. The propagation probability itself is a fast increasing function of the induction field, and all indications are that it only appears above a field threshold value, around $5 \mathrm{kV} \mathrm{cm}^{-1}$ in standard operating conditions. Since both discharge and propagation probability depend on the source ionization density, confirmation of the safety of operation of the detector in a given experiment can only be acquired with exposures in realistic conditions.

It is observed that at the onset of discharges only a small fraction of the ionizing events induce the transition; it might be questioned if this is due to statistical fluctuations within normal avalanches, or to some specific sub-class of events with low probability. Natural candidates for the second possibility are tracks releasing ionization within the high field regions normally associated with metal edges; in the GEM detectors, this is the region around the rims of the holes on the cathode side, where fields exceed the average by several orders of magnitude. An avalanche starting in this region can reach a much higher size and induce the transition; a similar process leading to discharges in micro-strip chambers, by spontaneous emission of electrons from cathode edges, has been described in Ref. [32]. Using a well-collimated alpha source, perpendicular to the detector, it should be, in principle, possible to disentangle the two processes, measuring discharge probability as a function of position in identical operating conditions. Alternatively, one could compare detectors having similar gain but different geometry, taking into account that the ratio between the normal multiplying field and the one on cathode edges depends on the diameter of holes and foil thickness.

The probability of discharge propagation through the induction gap and backwards to the drift electrode is strongly affected by the field strength and is mediated by the charge flow. Propagation between GEMs seems instead to be mainly due to the emission and re-absorption of photons in the gas or on the metallic surface of the electrodes. In some gases, the maximum gain that can be attained in presence of heavily ionizing radiation depends on the separation between GEMs, implying the presence of an effective photon feedback mechanism.

\section{Summary and conclusions}

In a systematic study of single and multiple GEM detectors, exposed to heavily ionizing particles, we have measured gains and probability of discharge formation and propagation in a wide range of operating conditions and gases. Compared to single devices, multiple cascaded structures permit to reach gains increasing by about an order of magnitude at each addition. Full discharge propagation, all the way from the multipliers to the read-out plane, only occurs above a critical value of the induction field, typically above $5 \mathrm{kV} \mathrm{cm}^{-1}$. A general trend to withstand higher gains in poorly quenched mixtures of argon and neon with isobutane has been found, counterbalanced by an expected loss of performance due to the larger size of the detected charge distribution and longer collection time. Moreover, these mixtures are flammable, and there are serious concerns about aging of the detectors due to gas polymerization at high rates.

Further studies aiming at uncovering positional or charge density dependence of the discharge probability may shed more light on the underlying physical processes involved, and indicate ways to improve the detectors to guarantee a reliable use in harsh experimental environments.

\section{References}

[1] F. Sauli, Nucl. Instr. and Meth. A 386 (1997) 531.

[2] A. Bressan, M. Hoch, P. Pagano, L. Ropelewski, F. Sauli, S. Biagi, A. Buzulutskov, M. Gruwé, A. Sharma, D. Moermann, G. De Lentdecker, Nucl. Instr. and Meth. A 424 (1999) 321.

[3] A. Bressan, L. Ropelewski, F. Sauli, D. Mörmann, T. Müller, H.J. Simonis, Nucl. Instr. and Meth. A 425 (1999) 254.

[4] T. Zeuner, Nucl. Instr. and Meth. A 446 (2000) 324. 
[5] S. Bachmann, A. Bressan, B. Ketzer, M. Deutel, L. Ropelewski, F. Sauli, Optimization of the gas electron multiplier for high rate applications, 8th Pisa Meeting on Advanced Detectors, Isola d'Elba, Italy, 21-27 May 2000, Nucl. Instr. and Meth. A 461 (2001) 42.

[6] M. Ziegler, P. Sievers, U. Straumann, Atriple GEM detector with two-dimensional readout, $\mathrm{LHCb}$ Int. Note 2000-056 (2000).

[7] P. Vanlaer, J.-M. Brom, J. Coffin, W. Geist, J.-M. Helleboid, A. Lounis, C. Maazouzi, G. Schuster, M.-H. Sigward, A. Zghiche, Y. Benhammou, F. Charles, J.-C. Fontaine, D. Huss, F. Jeanneau, A. Pallarès, Study of discharges in a large wedge-shaped MSGC+GEM detector exposed to a high intensity beam of pions at $300 \mathrm{MeV} / \mathrm{c}$, CERN-CMS Int. Note 1999/024, 1999.

[8] A. Bressan, J.C. Labbé, P. Pagano, L. Ropelewski, F. Sauli, Nucl. Instr. and Meth. A 425 (1999) 262.

[9] S. Bachmann, A. Bressan, A. Placci, L. Ropelewski, F. Sauli, IEEE Trans. Nucl. Sci. NS-47 (2000) 1412.

[10] S. Bachmann, A. Bressan, B. Ketzer, M. Deutel, L. Ropelewski, F. Sauli, A. Bondar, A. Buzulutskov, L. Shekhtman, A. Sokolov, A. Tatarinov, A. Vasil'ev, Performance of GEM detectors in high intensity particle beams, CERN-EP/2000-116, Nucl. Instr. and Meth. A 470 (2001) 548 .

[11] Y. Benhammou, R. Blaes, J.M. Brom, F. Drouhin, J.C. Fontaine, D. Huss, F. Jeanneau, V. Mack, I. Ripp-Baudot, B. Schwaller, A. Zghiche, Nucl. Instr. and Meth. A 441 (2000) 452.

[12] J.P. Perroud, Nucl. Instr. and Meth. A 453 (2000) 159.

[13] C. Iacobaeus, A. Brahme, M. Danielsson, P. Fonte, J. Ostling, V. Peskov, M. Wallmark, A novel portal imaging device for advanced radiation therapy, IEEE Nucl. Sci. Symposium (Lyon, October 15-20. 2000). IEEE Trans. Nucl. Sci., submitted for publication.

[14] M. Li, M. Dixit, J. Dubeau, P.J. Johns, Photon-counting digital radiography using high-pressure xenon filled detectors, Imaging 2000, Stockholm, June 28-July1, 2000, Nucl. Instr. and Meth. A 471 (2001) 215.

[15] D. Pacella, G. Pizzicaroli, L. Gabellieri, M. Leigheb, R. Bellazzini, A. Brez, G. Gariano, L. Lantronico, N. Lumb, G. Spandre, M.M. Massai, S. Reale, Ultrafast soft X ray 2D plasma imaging system based on the Gas Electron Multiplier detector with pixel read-out, 2000. Rev. Sci. Instrum. 72 (2001) 1372.

[16] P. Fonte, V. Peskov, B.D. Ramsey, Nucl. Instr. and Meth. A 419 (1998) 405.
[17] V. Peskov, B.D. Ramsey, P. Fonte, IEEE Trans. Nucl. Sci. NS-45 (1998) 244.

[18] Y. Ivanouchenkov, P. Fonte, V. Peskov, B.D. Ramsey, Nucl. Instr. and Meth. A 422 (1998) 300.

[19] P. Fonte, V. Peskov, B. Ramsey, IEEE Trans. Nucl. Sci. NS-46 (1999) 91.

[20] R. Bellazzini, M. Bozzo, A. Brez, G. Gariano, L. Latronico, N. Lumb, M.M. Massai, A. Papanestis, R.R.G. Spandre, M.A. Spezziga, Nucl. Instr. and Meth. A 425 (1999) 218.

[21] A. Mykulyak, H. Schmidt, H. Sann, H. Stelzer, Response of a double GEM structure to slow alpha particles, CERN ALICE Int. Note 99-55, 1999.

[22] V. Peskov, P. Fonte, M. Danielsson, C. Iakobauses, J. Ostling, M. Wallmark, The study and optimization of new micro-pattern detectors for high rate applications, IEEE Nuclear Science Symposium, Lyon, October 15-20, 2000, IEEE Trans. Nucl. Sci., submitted for publication.

[23] H. Raether, Electron Avalanches and Breakdown in Gases, Butterworth, London, 1964.

[24] G. Battistoni, P. Campana, V. Chiarella, U. Denni, B. Dettorre-Piazzoli, E. Iarocci, G. Mannocchi, G. Nicoletti, P. Picchi, Nucl. Instr. and Meth. 217 (1983) 433.

[25] F.E. Taylor, Nucl. Instr. and Meth. A 289 (1989) 283.

[26] B. Schmidt, Nucl. Instr. and Meth. A 419 (1998) 230.

[27] J. Benlloch, A. Bressan, M. Capeáns, M. Gruwé, M. Hoch, J.C. Labbé, A. Placci, L. Ropelewski, F. Sauli, Nucl. Instr. and Meth. A 419 (1998) 410.

[28] S. Bachmann, L. Bressan, L. Ropelewski, F. Sauli, A. Sharma, D. Mörmann, Nucl. Instr. and Meth. A 438 (1999) 376.

[29] B. Boimska, R. Bouclier, M. Capeáns, S. Claes, W. Dominik, M. Hoch, G. Million, L. Ropelewski, F. Sauli, A. Sharma, L. Shekhtman, W. Van Doninck, L. Van Lancker, Nucl. Phys. B 61B (1998) 498-503.

[30] J. Ostling, M. Wallmark, A. Brahme, M. Danielsson, C. Jacobaeus, P. Fonte, V. Peskov, Prog. Biomed. Opt. Imaging 3977 (2000) 84.

[31] R. Bouclier, W. Dominik, M. Hoch, J.C. Labbé, G. Million, L. Ropelewski, F. Sauli, A. Sharma, G. Manzin, Nucl. Instr. and Meth. A 396 (1997) 50.

[32] R. Bouclier, M. Capeáns, C. Garabatos, G. Manzin, G. Million, L. Ropelewski, F. Sauli, T. Temmel, L. Shekhtman, V. Nagaslaev, Y. Pestov, A. Kuleshov, Nucl. Instr. and Meth. A 365 (1995) 65. 